\title{
DEEP VEINS THROMBOSIS MIMICKING AN INFECTION IN A POSTPARTUM WOMAN
}

\author{
Entela Kolovani $^{1}$, Ergys Ramosaço $^{2}$, Edlira Bylykbashi ${ }^{3}$, Dhimiter Kraja ${ }^{4}$
}

\begin{abstract}
Ovarian Vein Thrombosis (OVT) is a rare, yet a serious complication, especially if it extends to an inferior cava vein. OVT can occur at any stage, but it mostly occurs during the postpartum period. Diagnosis is not often immediately apparent, and many other diseases can mimic this condition. Exclusion of any infectious etiologic cause in a postpartum patient with fever is the first step in establishing an OVT diagnosis. It can be accurately diagnosed by appropriate non-invasive investigations to enable early therapy with anticoagulants and intravenous antibiotics, which are the mainstay of treatment. We present a case of a 38-year-old female in the postpartum period who was diagnosed with right OVT reaching the inferior vein cava after a vaginal delivery. She was treated with intravenous antibiotic and anticoagulant therapy and had a successful recovery after repeated image six months after, showing complete resolution of thrombus.
\end{abstract}

UDC Classification: 618, DOI: https://doi.org/10.12955/pmp.v1.99

Keywords: Ovarian vein thrombosis, inferior cava inferior, postpartum,

\section{Introduction}

Ovarian Vein Thrombosis (OVT) is a rare postpartum, yet a serious complication. It can occur at any time, but mostly, it occurs during the postpartum period. Despite the imaging examinations performed in the emergency room, it is not always easy to diagnose because it is associated with non-specific symptoms.

Symptomatic OVT has an incidence reaching 1 in 2000-3000 deliveries, more often after cesarean delivery (Salomon et al., 2010; Rottenstreich et al. 2016). It may lead to other fatal complications such as sepsis, pulmonary emboli, or even death (Harun et al., 2014; Ortin Font. et al. 2005). A high index of suspicion is required to diagnose OVT in a patient with fever and abdominal pain during the postpartum period, notably in the right lower quadrant.

Prompt diagnosis and treatment of this condition are crucial in order to avoid unnecessary interventional procedures, morbidity, and mortality, all of them related to thrombosis, infections, or sepsis, which can associate OVT.

We present a case of a 38-year-old female in the postpartum period who was diagnosed with right OVT, complicated with inferior vein cava thrombosis. The patient did not report any previous history of thrombotic events.

The interest of this case we are presenting consists in the rarity of this entity, diagnose difficulties we faced, the attempts we made for the best treatment option of this puzzling clinical condition, and all the other problems we encountered in multidisciplinary collaboration among infectious diseases specialists, obstetricians, vascular surgeons, and haematologists.

\section{Material and methods}

We present a case of a postpartum patient with fever, complicated with Ovarian Vein Thrombosis extending in the inferior cava vein after a vaginal delivery. We conducted a systematic search where we included "pregnancy," "postpartum period," "ovary," "inferior cava vein", and " thrombosis" as keywords to the literature review.

\section{Case report}

In February 2019, a 38-year-old puerperal woman was admitted to our emergency room of Infectious Diseases Clinic for severe leg pain and ten days history of high fever with an immediate onset of 24 hours after an uncomplicated vaginal delivery. She had a history of four pregnancies and only two of them ended in childbirth.

\footnotetext{
${ }^{1}$ Infectious Diseases Clinic, University Hospital Centre "Mother Teresa” Tirana, Albania. vlasiovi@hotmail.com

${ }^{2}$ Infectious Diseases Clinic, University Hospital Centre "Mother Teresa" Tirana, Albania, erdara1er@yahoo.it

${ }^{3}$ University Hospital Obstetrics and Gynecology "Queen Geraldine", Tirana, Albania, eda.bylykbashi@gmail.com

${ }^{4}$ Infectious Diseases Clinic, University Hospital Centre "Mother Teresa" Tirana, Albania, lulikraja@hotmail.com
} 
The laboratory tests presented as follow: leukocyte count was $10.300 / \mu \mathrm{L}$, erythrocyte $4.0 \times 10^{6} / \mathrm{mm}^{3}, \mathrm{Hb}$ $11.6 \mathrm{~g} / \mathrm{dL}$, platelets $181.000 / \mu \mathrm{L}$, VES $27 \mathrm{~mm} / \mathrm{h}$, CRP $126 \mathrm{mg} / \mathrm{L}$ (reference range $0.8 \mathrm{mg} / \mathrm{L}$ to $5.0 \mathrm{mg} / \mathrm{L}$ ), CA-125 was $53 \mathrm{U} / \mathrm{mL}$ (normal value is less than $46 \mathrm{U} / \mathrm{mL}$ ). Urine analysis was normal. Blood and urine cultures showed no bacterial growth. Abdominal ultrasound and echocardiography resulted in normal. We started treatment with empiric ceftriaxone and ciprofloxacin for a possible infection. Unfortunately, the pain and fever did not resolve.

An abdominal Computed Tomography (CT) with intravenous contrast agent was performed on the third day of hospitalisation. It revealed a complete right ovarian vein thrombosis extending to the inferior cava vein with a $20 \%$ stenosis of its lumen, with a localisation below to the renal veins. (Fig 1,2). The chest computed tomography we performed excluded a pulmonary embolism.
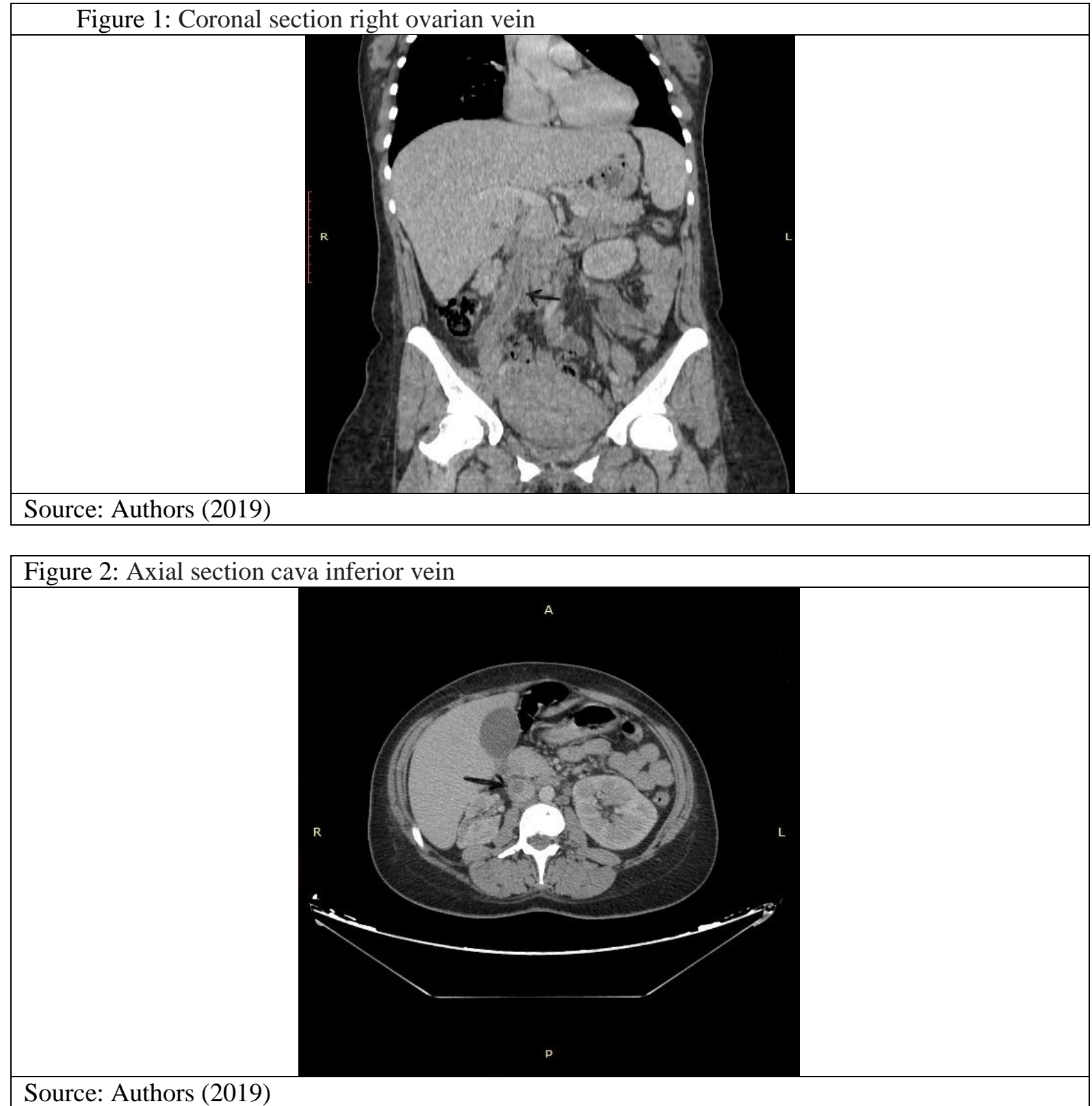

Anticoagulation therapy with enoxaparin 0.6 UI two times a day was initiated subcutaneously, associated with intravenous administration of imipenem, levofloxacin, and metronidazole. The patient's leukocytosis and fever resolved within 8 days. She made a good recovery and was discharged from our clinic after 20 days. After 9 months of follow-up, the patient remained free of symptoms. She continued anticoagulation treatment with rivaroxaban after discharge for six months, and now she has stopped medications and presents no recurrence of OVT. 


\section{Discussion}

Women are five times more likely to suffer from a thromboembolic event when they are pregnant. In a retrospective study of 74 cases of OVT, $81.1 \%$ of the cases were pregnancy-related (Rottenstreich et al., 2016). It can follow a term pregnancy, a premature delivery, an abortion, or an ectopic pregnancy.

Austin described the first case of OVT in the postpartum period in 1956 (Austin 1956). The estimated incidence of OVT ranges between 0.05 and $0.18 \%$ of pregnancies, with the majority of affected women being in the $3^{\text {rd }}$ or $4^{\text {th }}$ decade of their life (Kominiarek et al., 2006). In a prospective study, the incidence of OVT was $0.02 \%$ for vaginal deliveries and $0.1 \%$ for cesarean births. Multiparity has been identified as a risk factor for thrombosis (Ortin Font et al., 2004). The incidence peaks around day 2 postpartum for full-term deliveries and occurs within 10 days postpartum in $90 \%$ of cases (Klima et al., 2008).

We think that our patient is not that typical case to be complicated with OVT because she had an uncomplicated vaginal delivery. However, our patient had some risk factors for OVT, including postpartum period, age, and multiparity.

Regarding pathophysiology, OVT is explained by Virchow's triad: hyper-coagulation that is a pregnancy-related status, venous stasis due to compression of the inferior cava vein by the uterus, and endothelial trauma because of delivery or local inflammation. Pregnancy-related hypercoagulability is probably the most important risk factor. Protein $\mathrm{S}$ and fibrinolytic activity decreases, and factors V, VII, VIII, IX, and X, platelet count, and fibrinogen increases during the postpartum period (Calderwood et al., 2007).

Blood flow is retrograde in the left and anterograde in the right ovarian veins favouring the bacterial infections on this side. Another cause is that anatomically the right ovarian vein crosses in front of the ureter at the level of the L4 vertebra, consequently with right ureteral obstruction and hydronephrosis. Therefore, in most of the patients, uterine infection coexists with OVT, and this leads in a delay or misdiagnoses of OVT, which prevents the patient from the proper treatment.

The physiological dextrorotation of the uterus compresses the right ovarian vein. Since the right ovarian vein is longer than the left one and the venous stasis develops, these are the reasons why in $80-90 \%$ of cases, the right ovarian vein is affected (Savader et al., 1988; Greer et al., 2001).

Factors like: ovarian vein diameter that nearly triples its size by increasing blood flow, multiple incompetent valves that are present in the right ovarian vein, and hormonal changes that happen during pregnancy, resulting in substantially increased pressure on the vessel walls and the valves within the veins. This increased pressure at the valves is the leading cause of pelvic venous stasis (Kearon 2003; Scarsbrook et al., 2006).

Clinical symptoms are not specific for OVT. It is characterised by lower abdominal or flank pain, fever that usually appears during the first 10 days after delivery in $90 \%$ of cases (Derrick et al., 1997). Patients present with fever (80\%) and pain in the right iliac fossa (55\%), left lower quadrant (3.6\%) (Sorbi et al., 2016). Our patient presented to the clinic with complaints of severe leg pain and a fever of $39.3^{\circ} \mathrm{C}$ taking place 24 hours after her delivery.

Since the symptoms are not unique, the diagnosis of ovarian vein thrombosis may be delayed, and further complications can accompany this. Asymptomatic OVT is more common and may be benign, with a $30 \%$ incidence of pelvic (iliac and ovarian) vein thrombosis. Other patients with OVT may be diagnosed incidentally, for example, in aetiology workup to pulmonary embolism (Quarello et al. 2004).

Diagnosis of OVT can be made by Doppler Ultrasonography (US), contrast-enhanced computerised tomography scan (CT), and magnetic resonance imaging angiography (MRI) (Bates et al., 2002). Doppler US can visualise the absence of blood flow and increased ovarian vein diameter. However, obesity, peripartum bowel, and abdomen gases can reduce the imaging quality. The effectiveness of Doppler Ultrasonography (US) to confirm the diagnosis of OVT is highly operator dependent, which should limit its role in establishing the initial diagnosis (Nagayama et al., 2002). Doppler Ultrasonography maybe more recommended in follow-up imaging in patients previously diagnosed with OVT.

CT is the diagnostic test of choice with sensitivity $78-100 \%$ and specificity $63-99 \%$, but it requires exposure to pelvic radiation and IV contrast, an important consideration during pregnancy or postpartum (Kubik-Huch et al., 1999). Magnetic resonance image is considered ideal for its sensitivity and lack of 
ionising radiation. Therefore, CT should be used as an initial technique due to its cost-effectiveness (Jenayah et al., 2015).

The CT-scan was a vital examination for our patient as it confirmed the diagnosis and demonstrated the extension of thrombi inside ICV. It revealed a complete right ovarian vein thrombosis (figure 1), extending to the inferior cava vein (figure 2), with a $20 \%$ stenosis of its lumen, inferior to renal veins. It also helped us to exclude pulmonary embolism.

In the event that this disease remained undiagnosed or the diagnosis was delayed, complications may appear. So, OVT could extend toward the inferior cava vein, renal vein, or iliofemoral vessels, which can lead to pulmonary arterial embolisation. Thrombus can extend to the inferior vena cava in $15 \%$ and the renal vein in 12\% of cases (Al-toma et al., 2003; Wysokinska et al., 2006). Pulmonary embolism occurs in 13.2-25\% of untreated OVT patients, with a mortality of 4-5\% and with an estimated 18 deaths per million pregnancies (Dunnihoo et al., 1991; Khlifi et al., 2010; Harris et al., 2012). Other complications include sepsis due to thrombophlebitis, ovarian abscess, ovarian infarction, uterine necrosis, or ureteral compression (Plastini et al., 2017).

There are no specific treatment strategies implicated in the treatment of OVT. But the treatment of choice of OVT is a combination of anticoagulation and antibiotics when indicated (Dougan et al., 2016). The duration of anticoagulation treatment is not specifically addressed; in some articles, the duration ranged from 11 days to 60 months. In the two largest cohorts study, a median duration of 3 months of anticoagulation was applied in patients with OVT (Salomon et al., 2010).

The surgical procedures, as the initial management of OVT, is controversial. However, some clinicians prefer these procedures for complicated cases associated with free-floating thrombosis, recurrent pulmonary emboli despite medical treatment and contraindication to anticoagulant use (Kearon 2003). These procedures include placement of an IVC Greenfield filter or hysterectomy and thrombectomy, or even ligation of IVC (Clarke et al., 1999; Roberts et al., 2019). These procedures are not always possible to carry out and not in every clinic. Sometimes, the vascular surgeons do not prefer to treat them. Recurrence of OVT is low in future pregnancies. However, anticoagulant prophylaxis is recommended only in patients with underlying hyper coagulopathy status.

Regarding our case, anticoagulation therapy with enoxaparin was initiated. This treatment continued even after the patient left the hospital. Intravenous administration of broad-spectrum antimicrobials (imipenem, levofloxacin, and metronidazole) for a two weeks duration were the antibiotics of choice. She made a good recovery and discharged from our clinic after 20 days. After a 1-month follow-up, the patient remained free of symptoms. She received rivaroxaban for 6 months, and now, during the followup, she is free from anticoagulation therapy and presents no recurrence of OVT.

Exclusion of an infectious aetiology in a postpartum patient with fever was of great importance to us. Fever of unknown origin that persists despite antibiotics is a predictive clue to the formation of thrombus in the postpartum period (Togan et al., 2015). We faced a careful treatment choice challenge successfully and also the matter of which speciality should be responsible for her attendance. The best university clinics in our country have little experience in such cases. Complications accompanying OVT and its prognosis were a great challenge to us. Besides, the collaboration with other specialists such as vascular surgeons and gynaecologists remained challenging to achieve.

In our opinion, the absence of standardised attending and treatment protocols of this complex pathology needs a greater multidisciplinary collaboration of infectious diseases specialists, obstetriciangynaecologist, haematologists, radiologist, and vascular surgeons.

\section{Conclusions}

Although ovarian vein thrombosis is uncommon, with a high index of suspicion, it should be included in the differential diagnosis of fever especially in any women in the postpartum period, which presents with fever and lower abdominal pain. The disease has serious complications if left untreated. Early diagnosis is not always easy to achieve because of non-specific symptoms and signs. Experience, which is not enough and complications which are often risky, make different specialists give up the case. This clinical case is an important example of the great need we have to collaborate with other specialists in order to establish a quick diagnosis and proper treatment. Despite facing all these difficulties, we managed to make in time the right diagnosis to start the right treatment, hence minimisings the lifethreatening risk of a postpartum mother. 


\section{References}

Al-toma A, Heggelman G.F, Kramer M.H.H (2003). Postpartum ovarian vein thrombosis: report of a case and review of the literature. Netherlands Journal of Medicine. Vol. 61, no.10: 334-336

Austin OG (1956). Massive thrombophlebitis of the ovarian vein thrombosis. Am J Obstet Gynecol.; 72:428-429

Bates S.M, Ginsberg J.S (2002). How we manage venous thromboembolism during pregnancy. Blood. 15.;100(10):34703478

Calderwood C.J. Jamieson R, Greer I.A (2007). Gestational related changes in the deep venous system of the lower limb on light reflection rheography in pregnancy and the puerperium. Clinical Radiology. Vol 62. No 12. 1174-1179

Clarke C.S, Harlin S.A (1999). Puerperal ovarian thrombosis with extension into vena cava. Am Surg .65:147-150

Dougan C, Phillips R, Harley I, et al., (2016). Postpartum ovarian vein thrombosis. 2016.Obstetr, Gynaecology. 18, 291-299

Derrick FC Jr, Rosenblum RR, Lynch KM Jr (1997). Pathological association on the right ureter and right ovarian vein. J Urol, 97:633-640

Dunnihoo D.R, Gallaspy R.B, Wise R.B et al., (1991). Postpartum ovarian vein thrombophlebitis: a review. Obstetr and Gynecol Survey.Vol. 46, no. 7: 415-427

Greer. I.A. Thomson A. J (2001). Management of venous thromboembolism in pregnancy. Best Pract Res Clin Obstetr Gynaecol.;15:583-603

Harris K, Mehta S, Iskhakov E, et al., (2012). Ovarian vein thrombosis in nonpregnant women: an overlooked diagnosis. Ther Adv Hematol,3:325-328

Harun A, Sibel A, Sebahattin C et al., (2014). Postpartum ovarian vein and inferior vena cava thrombosis. Case Reports in Medicine. http://dx.doi.org/10.1155/2014/609187

Jenayah A.A, Saoudi S, Boudaya F, et al (2015). Ovarian vein thrombosis. Pan Afr Med J. 21:251

Kearon C (2003). Diagnosis of pulmonary embolism. CMAJ. Jan 168: 183-194

Klima D.A, Snyder T.E (2008). Postpartum ovarian vein thrombosis. Obstetr, Gynaecology.111. 431-435

Khlifi A, Kebaili S, Hammami M, et al., (2010). Postpartum ovarian vein thrombophlebitis: report of a case and review of the literature. North Am J Med Sci,2:389-391

Kominiarek MA, Hibbard JU (2006). Postpartum ovarian vein thrombosis: an update. Obstet Gynecol Surv.;61:337-342.

Kubik-Huch RA, Hebisch G, Huch R, et al., (1999). Role of duplex colour Doppler ultrasound, computed tomography, and MR angiography in the diagnosis of septic puerperal ovarian vein thrombosis. Abdominal Imaging.24(1).85-91

Nagayama M, Watanabe Y, Okumura A et al., (2002). Fast MR imaging in obstetrics. Radiographics. 22(3):563-580

Ortin Font X, Ugarriza A, Espax R. M. et al (2005). "Postpartum ovarian vein thrombosis ». Thrombosis and Haemostasis. Vol 93. No.5. 1004-1005

Plastini T, Henry D, Dunleavy K (2017). Ovarian vein thrombosis: to treat or not to treat? Blood Avd. 1:1120-1123

Quarello E, Desbriere R, Hartung O, et al., (2004). Postpartum ovarian veins thrombophlebitis: report of 5 cases and review of the literature. J Gynecol Obstet Biol Repro. 33:430-440

Roberts S. Ch, Dunn A. M (2019). Postpartum inferior vena cava thrombosis treated by endovascular suction thrombectomy utilizing veno-venous extracorporeal circulation. Journal Baylor University Medical Center. Volume 32, Issue 4, 554-556

Rottenstreich A, Da'as N, Kleinstern G at al (2016). Pregnancy and non-pregnancy related ovarian vein thrombosis: Clinical course and outcome. Thromb Res.;146;84-88

Salomon O, Dulitzky M, Apter S. (2010). New observations in postpartum ovarian vein thrombosis: experience of single center. Blood Coagul Fibrinolysis;21 (1):16-19

Savader S.J, Otero R.R, Savader L (1988). Puerperal ovarian vein thrombosis: Evaluation with CT, US, and MRI. Radiology, vol 167. No 3. 637-639

Scarsbrook A.F, Evans A.L, Owen A.R, Gleeson F.V (2006). Diagnosis of suspected venous thromboembolic disease in pregnancy. Clin Radiology. Jan 61:1-12

Sorbi F, Mannini L, Aldinucci M, et al., (2016). Ovarian Vein Thrombosis Presenting as Acute Abdomen in Puerperium. $J$ ClinDiagn Res.;10(2): QD03-4.

Togan T, Turan H, Cifci E, Çiftci C (2015). Ovarian and Renal Vein Thrombosis: A Rare Cause of Fever Outer the Postpartum Period. Case Rep Obstet Gynecol.:817862.

Wysokinska E.M, Hodge D, Mc Bane II R.D (2006). Ovarian vein thrombosis: Incidence of recurrent venous thromboembolism and survival, Thrombosis and Haemostasis, vol. 96, no.2, pp:126-131 